\title{
An analysis of the M31 microlensing events
}

\author{
Philippe Jetzer*t' \\ Institute of Theoretical Physics, University of Zurich, Winterthurerstrasse 190, CH-8057 Züurich \\ E-mail: jetzer@physik.uzh.ch
}

\begin{abstract}
We discuss the results obtained so far by microlensing towards the Andromeda galaxy. In particular, we focus on the events detected by the POINT-AGAPE and MEGA collaborations. The POINT-AGAPE collaboration concluded that at least $20 \%$ of the halo mass in the direction of M31 should be in the form of MACHOs, whereas the MEGA collaboration, although finding more events, claimed that their result is compatible with all events being due to self-lensing. We consider the spatial and time-duration distributions of the microlensing events for several mass distribution models of the M31 bulge. We find that only for extreme models of the M31 luminous components it is possible to reconcile the total observed MEGA events with the expected selflensing contribution. Moreover, the expected spatial distribution of self-lensing events is more concentrated and hardly agrees with the distribution observed by the MEGA collaboration. Nevertheless, the small number of events at disposal so far does not allow to draw firm conclusions on the halo dark matter fraction in the form of MACHOs.
\end{abstract}

The Manchester Microlensing Conference: The 12th International Conference and ANGLES Microlensing Workshop

January 21-25 2008

Manchester, UK

\footnotetext{
* Speaker.

${ }^{\dagger}$ This talk is based on work done in collaboration with G. Ingrosso, S. Calchi Novati, F. De Paolis, A.A. Nucita, G. Scarpetta and F. Strafella.
} 


\section{Introduction}

Since the proposal by Paczyński [1] gravitational microlensing has proved to be an efficient tool for studying the MACHO contribution to the dark matter in the halos of the Milky Way and of M31, the latter one as first discussed in Refs. [2], [3], and [4].

The interpretation of the results obtained so far remains, however, debated and controversial. Along the line of sight towards the LMC the MACHO collaboration, [5] reported a halo fraction of about $20 \%$ in the form of MACHOs with mass $\simeq 0.5 M_{\odot}$, while the latest results of the EROS collaboration towards both the SMC and the LMC are even compatible with a no MACHO hypothesis $[6,7]$. The case towards M31 is complicated by the fact that sources at baseline are unresolved, a situation referred to as "pixel-lensing" [2, 3, 8]. Still, a handful of microlensing events have been observed in the meantime [9-16]. The POINT-AGAPE collaboration made a high-threshold analysis of 3 years of data and found 6 bright short-duration microlensing events, one of which is a likely candidate for a binary lens event with caustic crossing [17]. A detailed analysis, including the determination of the efficiency, lead to the conclusion that the POINT-AGAPE results are compatible with the ones of the MACHO group with a lower limit on the halo fraction in the form of MACHOs of $\sim 20 \%$ for objects in the mass range $0.5-1 M_{\odot}$ (see Fig. 1). In contrast, the MEGA collaboration [18] finds that their results, although not conclusive, agree with a no MA$\mathrm{CHO}$ hypothesis. Although the issues involved in the microlensing observations towards the LMC or the M31 are indeed rather different, the results for the halo fraction in the form of MACHOs depend crucially on the prediction of the expected signal due to known luminous populations, this being dominated by the "self-lensing" signal where both source and lens belong to the same star population residing respectively either in the LMC or in M31.

The expected microlensing signal towards M31 has been discussed in a few works [19-22]. In this respect the modeling of the M31 luminous components is essential in order to get meaningful results. We explored these issues in detail $[23,24]$ taking into account the latest MEGA results. In particular, we computed the predicted number of events, which we then compared with the observational results and addressed also the question of whether the expected self-lensing signal due to stars belonging either to the bulge or the disc of M31 is able, as claimed by [18], to fully explain the MEGA results.

\section{Mass distribution in M31}

An important ingredient is the choice of a suitable mass model for M31, as discussed in detail in [24]. The visible mass distributions for the M31 bulge and disk are derived by fitting the observed brightness profiles given by [25] and by further assuming mass-to-light ratios for bulge and disk stellar populations. Moreover, the consideration of the M31 rotation curve data allows to derive the distribution of the dark matter in the M31 halo.

\subsection{M31 bulge}

The M31 bulge model is derived from Table I in [25] containing the bulge 3-D brightness density in the Gunn $r$-band and the ellipticity $\varepsilon(a)$ as a function of the major-axis distance $a$ to the M31 centre. The 3-D brightness profile is fitted with a single de Vaucouleurs $a^{1 / 4}$ law with central 
3-D brightness density $j_{r}(0)=9.57 \times 10^{-7} L_{\odot} \operatorname{arcsec}^{-3}$. This model accurately fits Kent data for $a_{\text {min }} \simeq 1$ arcmin, namely in the region usually explored by pixel lensing observations.

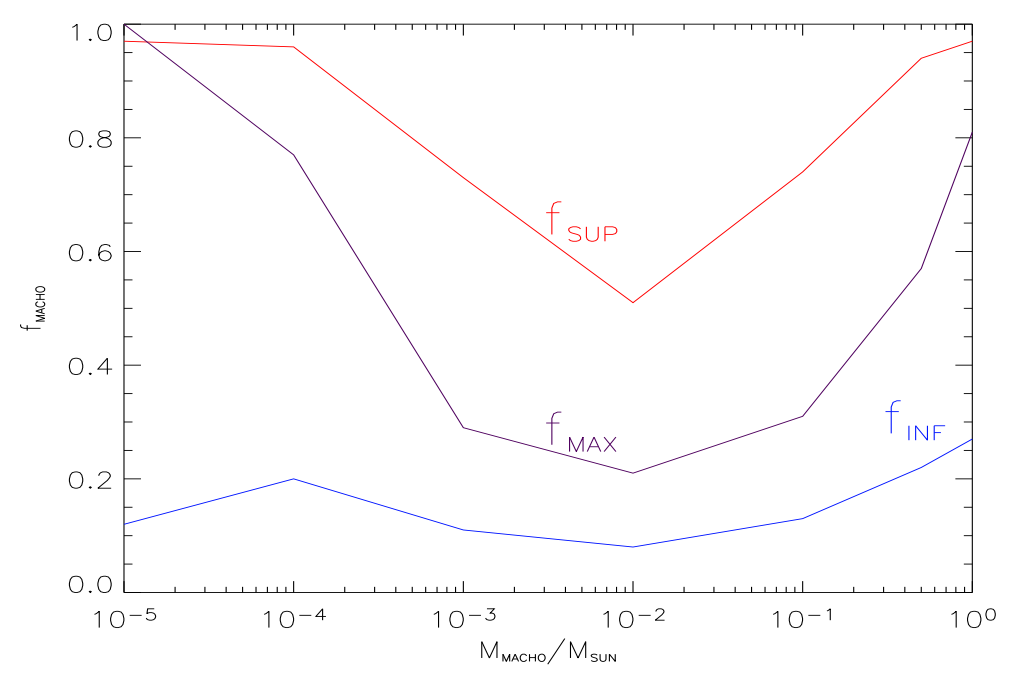

Figure 1: Most probable value, upper and lower 95\% CL limit for the halo mass fraction as a function of MACHO mass (from [17]).

From the 3-D brightness density profile one can then derive the corresponding mass density profile, which has the same behaviour as the brightness profile and central mass density given by

$$
\rho(0)=\left(\frac{M}{L_{R}}\right) 10^{-0.4\left[15.048-(r-R)-\mathrm{ext}_{R}-M_{\odot R}-d_{\mathrm{mod}}\right]},
$$

where $\left(M / L_{R}\right)$ is the mass-to-light ratio in the $R$-band, $(r-R)$ the colour of the bulge stellar population, $M_{\odot R}=4.42$ the absolute brightness of the Sun in the $R$-band, ext $_{R}$ the extinction in the same filter, and distance modulus $d_{\text {mod }}=24.43$ (for an M31 distance of $770 \mathrm{kpc}$ ). By using the values $\left(M / L_{R}\right)=2.96,(r-R)=0.59$ and $\operatorname{ext}_{R}=0.36$ quoted by [21], one obtains $\rho(0)=4.53 \times 10^{4} M_{\odot} / \mathrm{pc}^{3}$, corresponding to a total bulge mass $M_{\text {bulge }} \simeq 3.85 \times 10^{10} M_{\odot}$, in agreement with the value given by [25].

The observed 2-D brightness profile is also compatible with more concentrated mass distributions for the bulge [28]. As an example we considered a boxy model with $99 \%$ of the mass inside 4 kpc [24]. For comparison we also take into account the results obtained by using the bulge model adopted by the WeCapp collaboration [21].

\subsection{M31 Disk}

As in [19] the disk 3-D brightness density in the $r$-band is modeled by the law

$$
j_{r}(x, y, z)=j_{r}(0) \exp \left(-\sqrt{x^{2}+y^{2}} / h\right) \operatorname{sech}^{2}(z / H),
$$

and a best-fit procedure to the Kent data (for $a>6$ arcmin) allows to obtain the central brightness density $j_{r}(0)=4.2 \times 10^{-13} L_{\odot} \operatorname{arcsec}^{-3}$ (corresponding to a central magnitude $m_{r}(0)=20.5$ ), the radial scale length $h=27.95$ arcmin and the vertical scale length $H=1.34$ arcmin (corresponding to $h=6.4 \mathrm{kpc}$ and $H=0.3 \mathrm{kpc}$, respectively). 
As for the bulge, the corresponding disk mass density profile follows the same behaviour as the brightness profile. Accordingly, the disk central mass density is derived by assuming the following parameter values $\left(M / L_{R}\right)=0.88,(r-R)=0.54$ and $\operatorname{ext}_{R}=0.68$ for the disk [21], implying $\rho(0)=0.2 M_{\odot} \mathrm{pc}^{-3}$ and a total disk mass $M \simeq 3.09 \times 10^{10} M_{\odot}$.

\subsection{M31 and MW halos}

Both M31 and MW halo mass distributions are modeled as isothermal spheres

$$
\rho(r)=\frac{\rho_{0}}{1+\left(\frac{r}{r_{0}}\right)^{2}} .
$$

For M31 a fit to the M31 rotational curve by using the three-component model (bulge, disk, and halo) allows us to get the best-fit parameter values $r_{0}=2 \mathrm{kpc}$ and $\rho(0)=0.23 M_{\odot} \mathrm{pc}^{-3}$.

The overall M31 rotational curve which we obtain [24] reproduces well the data points derived from HI measurements of [26] and [27]. In comparison with the recent determination of the mass distribution in M31 [27], we find that at $R=35 \mathrm{kpc}$ the dark matter mass is $M_{\mathrm{h}}=3.7 \times 10^{11} M_{\odot}$ and the stellar mass $M_{\text {vis }}=6.6 \times 10^{10} M_{\odot}$. This translates to a total dynamical mass of $\simeq 4.4 \times 10^{11} M_{\odot}$ and to a rotational velocity of $233 \mathrm{~km} \mathrm{~s}^{-1}$ at $R=35 \mathrm{kpc}$, in agreement with the recent observations, and the M31 halo is truncated at $R=150 \mathrm{kpc}$.

For the MW we use a core radius $a \simeq 5.6 \mathrm{kpc}$ and a local $\left(R_{0}=8.5 \mathrm{kpc}\right)$ dark matter density $\rho\left(R_{0}\right) \simeq 1.09 \times 10^{7} M_{\odot} \mathrm{kpc}^{-3}$. The corresponding asymptotic rotational velocity is $v_{\text {rot }} \simeq 220 \mathrm{~km}$ $\mathrm{s}^{-1}$. The MW halo is truncated at $R \simeq 100 \mathrm{kpc}$.

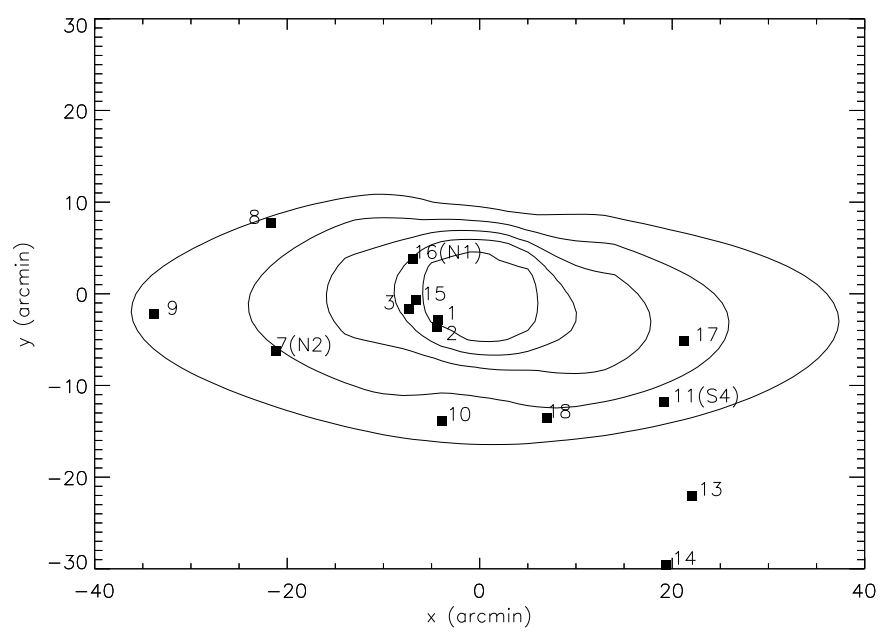

Figure 2: The map $d N_{\mathrm{ev}} / d \Omega$ of the expected (total) event rate towards M31 is shown, assuming the model discussed in the text, a MACHO mass value $\mu_{\mathrm{h}}=0.5$ and a MACHO halo dark matter fraction $f_{\mathrm{h}}=0.2$ (see text for details) (from [24]).

\subsection{Velocity dispersions}

The random velocities of stars and MACHOs are assumed to follow Maxwellian distributions, with one-dimensional velocity dispersion $\sigma=140$ and $166 \mathrm{~km} \mathrm{~s}^{-1}$ for the M31 bulge and MACHOs, and $\sigma=156 \mathrm{~km} \mathrm{~s}^{-1}$ for the MACHOs in the MW halo. Moreover, following [29], the M31 
disk stars are assumed to have one-dimensional dispersion velocity decreasing towards the outer part from the central value $\sigma(r=0) \simeq 110 \mathrm{~km} \mathrm{~s}^{-1}$ to $\sigma(r=30 \mathrm{kpc}) \simeq 5 \mathrm{~km} \mathrm{~s}^{-1}$. In addition, a rigid rotational velocity of $40 \mathrm{~km} \mathrm{~s}^{-1}$ has been taken into account for the M31 bulge [19].

\section{Results and conclusions}

In the tables and figures, we assume for both the M31 and MW halos a MACHO halo dark matter fraction $f_{\mathrm{h}}=0.2$, as suggested by microlensing observations towards the Magellanic Clouds [5] and pixel-lensing observations towards M31 [17]. However, most results can be easily rescaled to other values of $f_{\mathrm{h}}$.

Assuming the model discussed above (which we denote as reference model) for the M31 mass distribution, the spatial distribution of the expected event rate is shown in Fig. 2. We also adopt the observational parameters of the MEGA collaboration: we consider $T_{\mathrm{obs}}=2 \mathrm{yr}$ and we account for the detection efficiency and maximum impact parameter as given by [18]. In particular, in Fig. 2, from the outer to the inner M31 region, contour levels correspond to the values $5 \times 10^{-3}, 1 \times$ $10^{-2}, 2 \times 10^{-2}, 3 \times 10^{-2}, 1 \times 10^{-1}$ event $\operatorname{arcmin}^{-2}$, respectively. From Fig. 2 one can see that dark-lensing makes an important contribution to pixel-lensing beyond the second (from the inner) iso-rate contour, namely beyond $\simeq 10$ arcmin from the M31 centre.

The expected number of self-lensing events inside the 8 MEGA fields is given in Table 1 for different source and lens populations. Here with the symbols b, d, and h we indicate sources and/or lenses in the M31 bulge, disk, and halo, respectively. Capital symbol $\mathrm{H}$ is used to indicate lenses in the MW halo. In any case, the first (second) symbol refers to the source (lens). From Table 1 one can see that, for all the considered models (reference, boxy and WeCapp), the total number of self-lensing events is roughly the same (within $15 \%$ ).

For the reference and boxy models, we note an increase in bulge-bulge events to compensate for a decrease in disk-bulge ones. This is expected to be due to the different concentrations of bulge mass for the two distributions. We also note the increase in the disk-bulge events in the WeCapp model due to the more extended bulge mass distribution.

Table 1: Number of self-lensing events expected given the setup of the MEGA campaign, for different models (see text). We consider different source and lens populations.

\begin{tabular}{|c|c|c|c|c|c|}
\hline $\begin{array}{c}\text { Events inside } \\
\text { the } 8 \text { MEGA } \\
\text { fields }\end{array}$ & & & & & \\
\hline & $\mathrm{bb}$ & $\mathrm{bd}$ & $\mathrm{db}$ & $\mathrm{dd}$ & self \\
\hline reference & 4.25 & 1.17 & 3.30 & 0.96 & 9.68 \\
\hline boxy & 5.14 & 1.10 & 2.76 & 0.95 & 9.95 \\
\hline WeCapp & 4.98 & 1.34 & 4.08 & 0.96 & 11.37 \\
\hline
\end{tabular}

Assuming the reference model and $f_{\mathrm{h}}=0.2$, in Table 2 we give our estimate of the expected number of dark-lensing events for several MACHO mass values: $\mu_{\mathrm{h}}=0.1,0.5$, and 1 (in solar units). We find that the total number of dark-lensing and self-lensing events turns out to be roughly the same. The total (self+dark+background) number of expected events is $\sim 23$ including $\sim 1$ 
Table 2: For the reference model, the expected number of dark-lensing events is given for $\mu_{\mathrm{h}}=0.1,0.5,1$ and $f_{\mathrm{h}}=0.2$.

\begin{tabular}{|c|c|c|c|c|c|c|}
\hline $\begin{array}{c}\text { Events inside } \\
\text { the } 8 \text { MEGA } \\
\text { fields }\end{array}$ & & & & & & \\
\hline & $\mu_{\mathrm{h}}$ & bh & $\mathrm{bH}$ & $\mathrm{dh}$ & $\mathrm{dH}$ & dark \\
\hline \multirow{3}{*}{ reference } & 0.1 & 2.55 & 1.04 & 8.81 & 3.10 & 14.49 \\
\hline & 0.5 & 1.96 & 0.72 & 6.85 & 2.23 & 11.76 \\
\hline & 1 & 1.68 & 0.58 & 5.80 & 1.82 & 9.88 \\
\hline
\end{tabular}

event due to supernovae ( $\mathrm{SN}$ ) contamination. This is consistent at a $2 \sigma$ confidence level with the 14 candidate MEGA events assumed to follow a Poisson distribution.

A comparison of our results with the corresponding values reported in Table 5 of [18] shows that there is fairly good agreement. Indeed, to get a more meaningful comparison for the selflensing contribution, we normalised the values for the mass of the luminous components to those of the MEGA models. We found that our estimate of the (total) number of the self-lensing events agrees with the [18] prediction only when considering more extreme (maximal) parameters for the disk component.

At variance with [18] we nevertheless do not conclude that all the 14 events detected by the MEGA collaboration can be explained only by self-lensing. Indeed, the spatial distribution of the events occurring inside the 8 MEGA fields clearly shows that the distribution with the distance from the M31 centre of the self-lensing events hardly can be reconciled with the MEGA data.

In Fig. 3, for the reference (dotted line) and boxy (dashed line) models, the (normalized) distribution of the expected number of self-lensing events within the 8 MEGA fields is given as a function of the the distance from the M31 centre. The same quantity is shown for self+dark lensing (thin solid line) assuming the reference model, $f_{\mathrm{h}}=0.2$ and $\mu_{\mathrm{h}}=0.5$. For comparison the (normalized) distribution of the 14 observed MEGA events is also given (thick solid line). An excess of events with respect to expectations from self-lensing remains at a large distance. This conclusion is enhanced when assuming the boxy model for the M31 bulge.

A better agreement with MEGA data can be obtained if one also considers a dark-lensing (with $\mu_{\mathrm{h}}=0.5$ and $f_{\mathrm{h}}=0.2$ ) contribution. The compatibility between the observed MEGA event distribution as a function of distance from the M31 centre and the expected one has been evaluated for both self-lensing and the self+dark lensing hypotheses. By using the Kolmogorov-Smirnov test, we find a K-S probability $\simeq 0.51$ for self+dark lensing and $\simeq 0.18$ for self-lensing only, thus implying that a dark matter contribution to microlensing seems to be favoured.

However, we caution that the candidate microlensing events could be contaminated by variable stars. In particular, the events labeled 13 and 14, located in a region where the microlensing rate is negligible, might be contaminated by background supernovae. Indeed, by assuming standard $\mathrm{SN}$ rate and integrating over the volume within $z_{\max } \simeq 0.4$ (the maximum distance at which the SN signal-to-noise ratio is at least $3 \sigma$ above the typical baseline of $22 \operatorname{mag~arcsec}^{-2}$ ), we expect about one detectable $\mathrm{SN}$ in the outer M31 regions during the observational MEGA campaign.

The distribution of the expected number of events with the time scale $t_{1 / 2}$ is shown in Fig. 4 for 


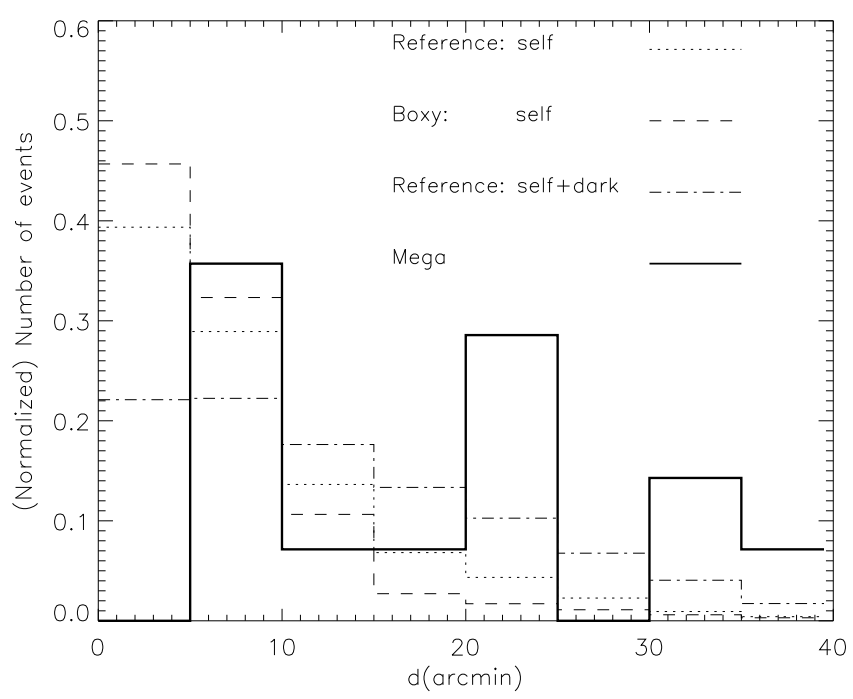

Figure 3: The normalised distribution of the expected number of self-lensing events within the 8 MEGA fields as a function of the the distance from the M31 centre (from [24]).

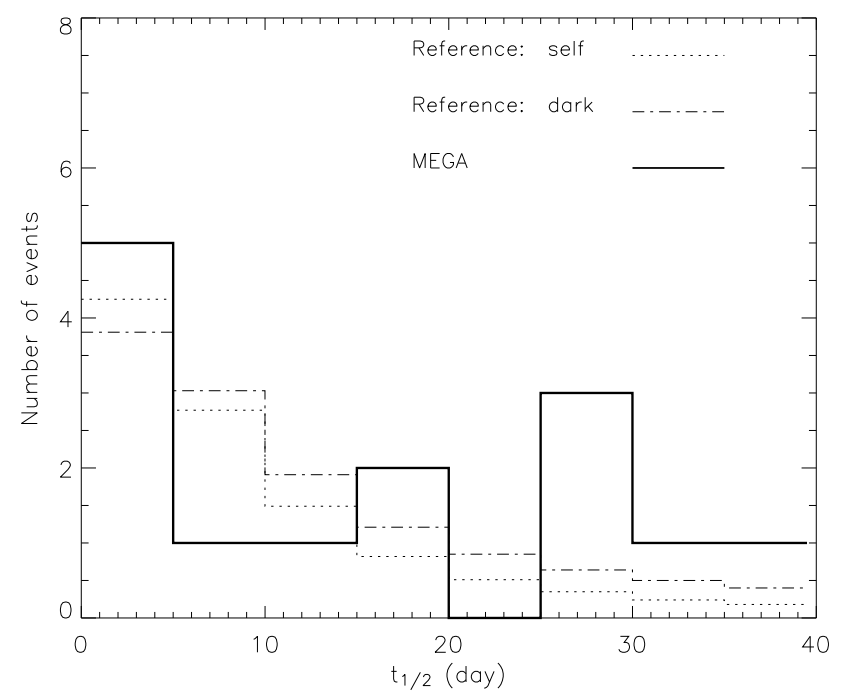

Figure 4: The expected event number within the $8 \mathrm{MEGA}$ fields as a function of $t_{1 / 2}$, for both self-lensing (dotted line) and dark-lensing (dot-dashed line) in the case of the reference model. For comparison the distribution with $t_{1 / 2}$ of the 14 observed MEGA events is also given (from [24]).

the reference model and $f_{\mathrm{h}}=0.2$, and $\mu_{\mathrm{h}}=0.5$. From this figure, one can see that self-lensing and dark-lensing events almost have the same $t_{1 / 2}$ distribution. Therefore, the $t_{1 / 2}$ event distribution is not particularly useful for determining the nature of the 14 MEGA events, at least for a MACHO mass value near $0.5 M_{\odot}$ (see also [23]). The excess of long duration events in the MEGA data also suggests a contamination by other variable objects.

We emphasize that our analysis shows that hardly any 14 MEGA events can be due to selflensing events by M31 stars. On the other hand, given the few events detected up to now, the estimate of the halo dark matter fraction in form of MACHOs given by POINT-AGAPE has to be taken as preliminary. 
I would like to thank S. Calchi Novati for carefully reading the manuscript and useful suggestions.

\section{References}

[1] B. Paczyński, 1986, ApJ 304, 1

[2] A.P.S. Crotts, 1992, ApJ 399, L43

[3] P. Baillon, A. Bouquet, Y. Giraud-Heraud \& J. Kaplan, 1993, A\&A 277, 1

[4] Ph. Jetzer, 1994, A\&A 286, 426

[5] C. Alcock,R.A. Allsman, D.R. Alves et al., 2000, ApJ 542, 281

[6] C. Afonso, J.N. Albert, J. Andersen et al., 2003, A\&A 400, 951

[7] P. Tisserand, L. Le Guillou, C. Afonso et al., 2007, A\&A 469, 387

[8] A. Gould, 1996, ApJ 470, 201

[9] M. Auriere, P. Baillon, A. Bouquet et al., 2001, ApJ 553, L137

[10] S. Calchi Novati, G. Iovane, A. Marino et al., 2002, A\&A 381, 848

[11] S. Calchi Novati, Ph. Jetzer, G. Scarpetta et al., 2003, A\&A 405, 851

[12] S. Paulin-Henriksson, P. Baillon, A. Bouquet et al., 2003, A\&A 405, 15

[13] A. Riffeser, J. Fliri, R. Bender et al., 2003, ApJ 599, L17

[14] J.T.A. de Jong, K. Kuijken, A.P.S. Crotts et al., 2004, A\&A 417, 461

[15] V. Belokurov, J. An, N.W. Evans et al., 2005, MNRAS 357, 17

[16] Y.C. Joshi, A.K. Pandey, D. Narasimha \& R. Sagar, 2005, A\&A 433, 787

[17] S. Calchi Novati, S. Paulin-Henriksson, P. Baillon et al., 2005, A\&A 443, 911

[18] J.T.A. de Jong, L.M. Widrow, P. Cseresnjes et al., 2006, A\&A 446, 855

[19] E. Kerins, B. Carr, N.W. Ewans et al., 2001, MNRAS 323, 13

[20] E.A. Baltz, G. Gyuk \& A.P.S. Crotts, 2003, ApJ 582, 30

[21] A. Riffeser, J. Fliri, S. Seitz, R. Bender, 2005, ApJS 163, 225

[22] E. Kerins, M.J. Darnley, J. Duke et al., 2006, MNRAS 365, 1099

[23] G. Ingrosso, S. Calchi-Novati, F. De Paolis et al., 2006, A\&A 445, 375

[24] G. Ingrosso, S. Calchi-Novati, F. De Paolis et al., 2007, A\&A 462, 895

[25] S.M. Kent, 1989, AJ 97, 1614

[26] E. Brinks \& W.N. Burton, 1984, A\&A 141, 195

[27] C. Carignan, L. Chemin, W.K. Huchtmeier and F.J. Lockman, 2006, ApJ 641, L109

[28] R.L. Beaton, S.R. Majewski, P. Guhathakurta et al., 2007, ApJ 658, L91

[29] L.M. Widrow \& J. Dubinski, 2005, ApJ 631, 838 